\title{
Growth, photosynthesis, and reactive oxygen system responses of allotriploid Populus cathayana to salt stress
}

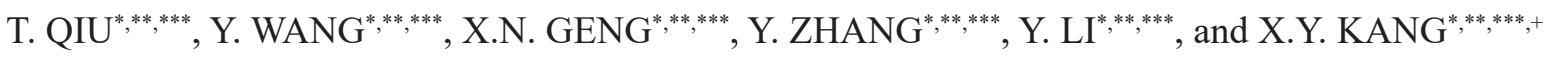 \\ Beijing Advanced Innovation Center for Tree Breeding by Molecular Design, College of Biological Sciences \\ and Technology, Beijing Forestry University, 100083 Beijing, China* \\ National Engineering Laboratory for Tree Breeding, Ministry of Education, Beijing Forestry University, \\ 100083 Beijing, China** \\ Beijing Laboratory of Urban and Rural Ecological Environment, College of Biological Sciences and Technology, \\ Beijing Forestry University, 100083 Beijing, China ${ }^{* * *}$
}

\begin{abstract}
Polyploid plants are generally considered to have a high stress resistance. In this study, two full sibling allotriploid populations (FDR and SDR) with different $2 \mathrm{n}$ female gamete sources and one diploid population (2N) obtained from the same Populus cathayana parents were investigated. We compared the growth morphology, photosynthetic parameters, peroxidase activity and proline contents after salt stress. The results showed that the salt tolerance of the triploid poplar population was higher than that of the diploid population. And there were some differences between the genotypes of the triploid and diploid populations under salt stress. The genotypes with the higher salt tolerance in the triploid populations were much more tolerant than those with the higher salt tolerance in the diploid populations. There was no significant difference in salt tolerance between the FDR and SDR triploids.
\end{abstract}

Additional key words: heterozygosity; malondialdehyde; photosynthetic efficiency; relative membrane permeability.

\section{Introduction}

It is generally believed that polyploid plants have a strong resistance to stress, such as saline-alkaline, cold, drought, and heat stress (Segraves and Anneberg 2016, Godfree et al. 2017, Luo et al. 2017). After doubling the chromosomes in the plant nucleus, due to the increase in the gene dose, the stress resistance of polyploid plants is enhanced by regulating the biomembrane system, improving the content of osmotic substances, and enhancing the activity of the antioxidant system (Zhang et al. 2015). Studies with Arabidopsis, wheat, and Robinia pseudoacacia have confirmed that polyploid plants show more resistance to high saline-alkaline environments than diploid plants (Chao et al. 2013, Yang et al. 2014, Meng et al. 2016). However, there have been contradictory research results for this topic. Sugiyama (2006) found that diploid wheat straw had higher survival and growth rates than tetraploid straw under drought stress. In some salt tolerance trials, some diploid buffalo grasses from different habitats were also more tolerant than their polyploid counterparts $(\mathrm{Wu}$ and Lin 1994). However, the results of studies on salt tolerance in polyploid Brassica were not consistent. Some studies have shown that polyploids are more salt tolerant, and some studies have found the opposite (He and Cramer 1992, Kumar 1995, Ashraf 2001). The reason may be related to the biological characteristics and the small sample size error caused by genotype differences.

In recent years, our research team has created a large number of triploid germplasm of poplars based on breakthroughs in sexual polyploidization (Wang et al. 2012). Then, we asked how does the stress resistance in triploid poplar compare with that in diploid poplar? To answer this question, a large sample size of two populations of allotriploid Populus cathayana (FDR and SDR) from different sources of $2 \mathrm{n}$ female gametes and a diploid $(2 \mathrm{~N})$ population with the same parents were selected for this study. The differences in physiological parameters, such as growth morphology, photosynthesis, and antioxidant enzyme activity under salt-stress treatment were compared

\footnotetext{
Received 1 November 2019, accepted 15 May 2020.

${ }^{+}$Corresponding author; phone: +86 010 62337708, e-mail: xykang@163.com

Abbreviations: $2 \mathrm{~N}$ - diploid population; $\mathrm{CCI}$ - chlorophyll content index; $C_{\mathrm{i}}-$ intercellular $\mathrm{CO}_{2}$ concentration; $\mathrm{D}$ - ground diameter; $E$ transpiration rate; FDR - allotriploid population; $g_{s}$ - stomatal conductance; $\mathrm{H}$ - plant height; LA - leaf area; MDA - malondialdehyde; PEw - photosynthetic efficiency of the whole leaf; $P_{\mathrm{N}}$ - net photosynthetic rate; POD - peroxidase; Pro - proline; RMP - relative membrane permeability; ROS - reactive oxygen species; SDR - allotriploid population; TCA - trichloroacetic acid.

Acknowledgements: The research was supported by National Natural Science Foundation of China of 'Molecular Basis of the Vegetative Growth Advantage in Allotriploid Poplar' (31530012) and the Program of the Co-Construction with Beijing Municipal Commission of Education of China.
} 
at the population level to lay a theoretical foundation for the physiological response mechanism of salt tolerance in polyploid plants.

\section{Materials and methods}

Plant materials: Test materials included two synthetic allotriploid $(2 \mathrm{n}=3 \mathrm{x}=57)$ and one diploid $(2 \mathrm{n}=2 \mathrm{x}=$ 38) progeny populations. Three populations have the same parents, namely, Populus pseudo-simonii $\times$ P. nigra 'Zheyin ${ }^{\#}$ ' and P. beijingensis. During different meiosis stages of megaspore mother cells, high temperature treatment can induce heterologous triploids with different $2 \mathrm{n}$ gametes. Then, the $2 \mathrm{n}$ female gamete produced by the first division restitution (FDR) and the normal pollen can undergo pollination hybridization to form the FDR triploid. Similarly, the $2 \mathrm{n}$ female gamete produced by the second division restitution (SDR) can be hybridized to the SDR triploid.

Salt stress treatment: There were FDR and SDR triploid and diploid (2N) Populus cathayana populations and 16 genotypes were randomly selected from each population. Each population had two treatments, namely, the untreated control group and the salt stress group. In March 2017, ten cuttings (approximately $15 \mathrm{~cm}$ in length, one-year old) of each genotype were cultivated in pots $(23-\mathrm{cm}$ upper diameter $\times 18-\mathrm{cm}$ lower diameter $\times 25-\mathrm{cm}$ depth) filled with soil (40\% clay, $40 \%$ sand, and $20 \%$ vermiculite). All plants were grown in a glasshouse (natural sunlight, temperatures of $24-28^{\circ} \mathrm{C}$, relative humidity of $60-80 \%$ ) in Beijing Forestry University, Beijing, China. Six healthy plants of about one-meter height for each genotype were used for the salt stress experiments in July 2017. We tested and analyzed three biological replicates with identical growth performances from each genotype in the different treatments. $\mathrm{NaCl}$ solution $(70 \mathrm{mM})$ was poured into the pots every three days, $1 \mathrm{~L}$ for each pot at every turn (Yang et al. 2009, Chen et al. 2012). After continuous treatment for $30 \mathrm{~d}$, we began to measure all plants $48 \mathrm{~h}$ after the last salt-water pouring, when the physiological indexes of the plants tended to be stable.

Phenotype and photosynthesis: After salt treatment for $30 \mathrm{~d}$, every eighth mature leaf from the top of the stem was sampled at 08:30-11:30 h. The net photosynthetic rate $\left(P_{\mathrm{N}}\right)$, stomatal conductance $\left(g_{\mathrm{s}}\right)$, transpiration rate $(E)$, and intercellular carbon dioxide concentration $\left(C_{\mathrm{i}}\right)$ were measured by an $L I-6400$ portable photosynthesis system (LiCor-6400, Li-Cor Inc., Lincoln, NE, USA) with the $\mathrm{CO}_{2}$ concentration at $400 \mu \mathrm{mol} \mathrm{mol}{ }^{-1}$, the PPFD at 1,400 $\mu \mathrm{mol}\left(\right.$ photon) $\mathrm{m}^{-2} \mathrm{~s}^{-1}$, the leaf temperature of $24 \pm 1^{\circ} \mathrm{C}$, and the relative air humidity of $60-65 \%$, leaf chamber of $2 \times 3 \mathrm{~cm}$. The chlorophyll $(\mathrm{Chl})$ content index (CCI) of the same leaf was measured using a CCM-200 Plus chlorophyll content meter (OPTI-Sciences Inc., USA). Morphological indexes of plant height $(\mathrm{H})$, ground diameter (D), and leaf area (LA) were measured. The photosynthetic efficiency of the whole leaf was calculated as PEw $=P_{\mathrm{N}} \times$ LA.
Reactive oxygen species (ROS): The production of ROS leads to membrane lipid peroxidation and the accumulation of osmoregulatory substances in cells to maintain their stability, while antioxidant enzymes are produced to remove ROS. The eighth leaf, which was cut into 20 discs $(0.8 \mathrm{~cm}$ in diameter), was used to determine the relative membrane permeability (RMP). Remaining leaves were weighed immediately after harvest to determine the fresh mass (FM). The initial electrical conductivity (EC1) was measured using a conductivity instrument (EC215, HANNA Instruments Co. Ltd., Italy) after placing the discs in deionized water for $2 \mathrm{~h}$ at $25^{\circ} \mathrm{C}$. The samples were boiled for $20 \mathrm{~min}$, after which they were cooled to $25^{\circ} \mathrm{C}$, and the second measurement of electrical conductivity (EC2) was obtained. RMP was calculated as follows: $\mathrm{RMP}[\%]=\mathrm{EC} 1 / \mathrm{EC} 2 \times 100$. The malondialdehyde $(\mathrm{MDA})$ content was measured by the improved thiobarbituric acid spectrophotometric method (Castrejón and Yatsimirsky 1997). A total of $0.5 \mathrm{~g}$ powdered leaf tissue was homogenized in $10 \mathrm{ml}$ of $10 \%(\mathrm{w} / \mathrm{v})$ trichloroacetic acid (TCA) and centrifuged at $8,000 \times g$ for $10 \mathrm{~min}$. Next, $2 \mathrm{ml}$ of $0.6 \%(\mathrm{w} / \mathrm{v})$ thiobarbituric acid in $10 \%$ TCA were mixed with $2 \mathrm{ml}$ of supernatant. The mixed solution was incubated in boiling water for $40 \mathrm{~min}$ and then cooled with running water. The mixture was centrifuged at $8,000 \times g$ for $10 \mathrm{~min}$, and the absorbance of the supernatant was measured at $532 \mathrm{~nm}$ using a spectrophotometer (Ultrospec 6300 pro, Cambridge, England). Peroxidase (POD, EC 1.11.1.7) was measured by the improved Wang et al. (2014) method. The powdered leaf tissue $(0.1 \mathrm{~g})$ was homogenized in $1 \mathrm{ml}$ of $50 \mathrm{mM}$ phosphate buffer $(\mathrm{pH}$ 7.2). The absorbance $\mathrm{A} 1$ at $470 \mathrm{~nm}$ for $30 \mathrm{~s}$ and $\mathrm{A} 2$ at $1 \mathrm{~min}$ for $30 \mathrm{~s}$ was recorded by using a spectrophotometer (Ultrospec 6300 pro, Cambridge, England). Activity was calculated as $\Delta \mathrm{A}=\mathrm{A} 2-\mathrm{A} 1$. The absorbance change 0.005 in a minute per gram of leaf tissue in 1-ml reaction system was defined as 1 unit of POD activity at $470 \mathrm{~nm}$. Proline (Pro) content was measured according to Vieira et al. (2010) with minor modifications. The powdered leaf $(0.1 \mathrm{~g})$ was homogenized in $1 \mathrm{ml}$ of $3 \%(\mathrm{w} / \mathrm{v})$ aqueous sulfosalicylic acid solution and then centrifuged at $10,000 \times g$ for $10 \mathrm{~min}$. The supernatant $(0.25 \mathrm{ml})$ reacted with $0.25 \mathrm{ml}$ of acid ninhydrin and $0.25 \mathrm{ml}$ of glacial acetic acid for $30 \mathrm{~min}$ at boiling temperature. The mixture was cooled and $0.5 \mathrm{ml}$ of toluene was added. The absorbance of the mixture was measured at $520 \mathrm{~nm}$ (Ultrospec 6300 pro, Cambridge, England). Pro content was calculated as $50,000 \times \Delta \mathrm{A}$ and expressed in $\mu \mathrm{g} \mathrm{g}^{-1}(\mathrm{FM})$.

Statistical analysis: All phenotypes and physiological indexes of the three populations were analyzed by the SPSS 20.0 software package (SPSS Inc., Chicago, IL, USA). Statistical differences were analyzed using one-way analysis of variance $(A N O V A)$ and the means for each treatment were compared by the Fisher's LSD test. The within-population coefficient of variation (CV) was calculated as the standard deviation (SD) divided by the mean. Prism 6.0 software (GraphPad Software, La Jolla, CA, USA) was used for the graphical analysis to repre- 
sent physiological diversity under salt stress between populations.

\section{Results}

Growth and morphology: The plant height $(\mathrm{H})$, ground diameter, and leaf area (LA) of the diploid (2N) and two allotriploid populations (FDR and SDR) were measured (Fig. 1; Table 1S, supplement). Under normal culture conditions, the average values of $\mathrm{H}$, ground diameter, and LA of the triploid plants were higher than those of the diploid plants. The average LA values of the FDR triploid population were slightly larger than those of the SDR triploid population, but the difference did not reach a significant level. After salt stress, the average ground diameter and LA values of the triploid were significantly higher than those of the diploid. The average LA of the FDR, SDR, and diploid plants was 78.85, 73.36, and $64.67 \mathrm{~cm}^{2}$, respectively. The average $\mathrm{H}$, diameter, and LA values of the FDR triploid population were greater than those of the diploid and SDR triploid populations, but there was not a significant difference.

In terms of the individual characteristics of the different populations, the genotypes showed some affect between the triploid and diploid poplar populations on plant $\mathrm{H}$, ground diameter, and LA without salt treatment, especially on LA. For the variations in plant $\mathrm{H}$ and

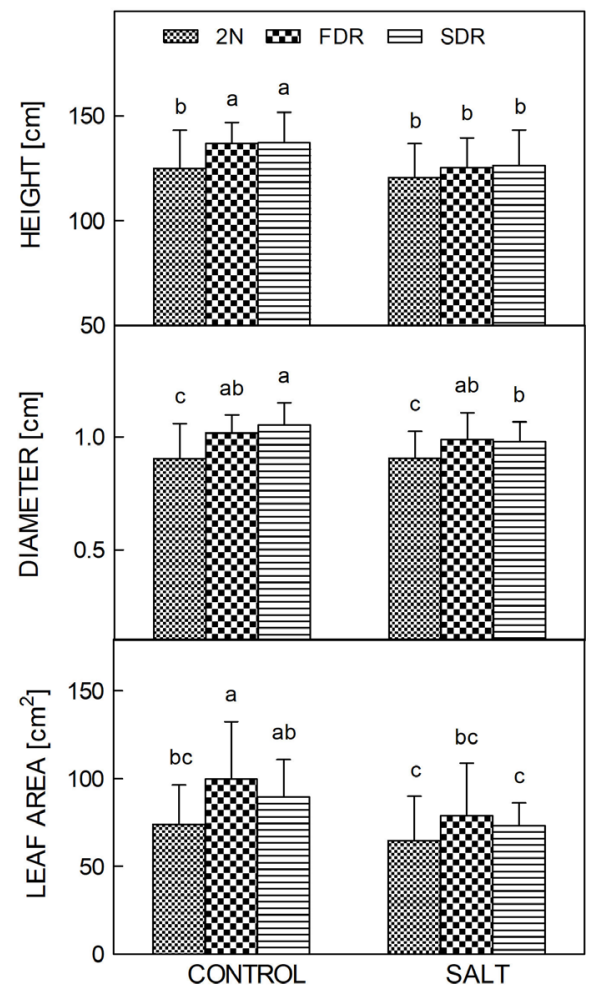

Fig. 1. Changes in height, ground diameter, and leaf area of the diploid $(2 \mathrm{~N})$ and two allotriploid populations (FDR and SDR) after $30 \mathrm{~d}$ of salt treatment. Mean values $\pm \mathrm{SD}(n=16)$. Values with the same letter are not significantly different according to the Fisher's LSD test $(p<0.05)$. ground diameter within the populations, the coefficients of variation of the FDR population were 0.07 and 0.08 , and for the SDR triploid population, they were 0.11 and 0.09 , respectively, which were lesser than those in the diploid populations. The coefficients of variation for plant $\mathrm{H}$ and ground diameter were 0.15 and 0.17 , respectively, for the diploid populations. For the variation in LA within the populations, the coefficients of variation of the FDR and SDR triploids were 0.33 and 0.24 , respectively, while those of the diploids were 0.30 . For the salt treatment group, the coefficients of variation of the growth indicators in FDR triploid population were larger than those of the control group. The coefficient of variation in the triploid population was lower than that in the diploid population. The LA coefficients of variation of the FDR and SDR triploids were 0.38 and 0.17 , respectively, while that of the diploids was 0.39 .

Photosynthesis: The average values of photosynthesisrelated traits in the different treatments are shown (Fig. 2; Table 2S, supplement). The average values of $P_{\mathrm{N}}, g_{\mathrm{s}}, C_{\mathrm{i}}$, and $E$ of all populations without salt treatment were not significantly different, but the average CCI of the triploid plants was significantly greater than that of the diploids. The average CCI values of the FDR and SDR triploids were 41.5 and $24.0 \%$, respectively, higher than those of diploids. Moreover, in terms of net photosynthetic efficiency, the average PEw value of the triploid poplar was greater than that of the diploid poplar. The average PEw values of the FDR and SDR triploids were 0.10 and $0.09 \mu \mathrm{mol} \mathrm{s}{ }^{-1}$, respectively, which were 31.1 and $15.3 \%$ higher than those of the diploids. There was no significant difference in $P_{\mathrm{N}}, g_{\mathrm{s}}, C_{\mathrm{i}}, E$, and PEw between the FDR and SDR triploids, but the average value of CCI of the FDR triploid was significantly higher than that of the SDR triploid. After $30 \mathrm{~d}$ of salt stress, the $P_{\mathrm{N}}, g_{\mathrm{s}}, E$, CCI, and PEw of the triploid and diploid populations were significantly lower than those of the untreated groups. There was significant difference in CCI between the diploid and triploid populations. In addition, the average PEw values of the FDR and SDR triploids were 0.054 and $0.048 \mu \mathrm{mol} \mathrm{s}^{-1}$, respectively, which were 25.4 and $11.1 \%$ higher than those of the diploids. There were no significant differences in the photosynthetic parameters between the FDR and SDR triploid after salt treatment.

From the individual traits of the different groups, the coefficients of variation of $P_{\mathrm{N}}, g_{\mathrm{s}}$, and $C_{\mathrm{i}}$ in the triploid population without salt treatment were higher than those in the diploid population, while the coefficients of variation of $E, \mathrm{CCI}$, and PEw in the triploid population were lower than those in the diploid population. After salt treatment, the coefficients of variation of the photosynthesis-related indexes in the different populations were all higher than those in the control group. The coefficients of variation of $P_{\mathrm{N}}$ and PEw in the triploids were higher than those in the diploids, while those of $g_{\mathrm{s}}, C_{\mathrm{i}}, E$, and CCI in the triploids were lower than those in the diploids. The coefficients of variation of PEw in the FDR and SDR triploids were 0.75 and 0.78 , respectively, and the variation ranged from $0.01-0.16 \mu \mathrm{mol} \mathrm{s}^{-1}$. The coefficient of variation of PEw in 


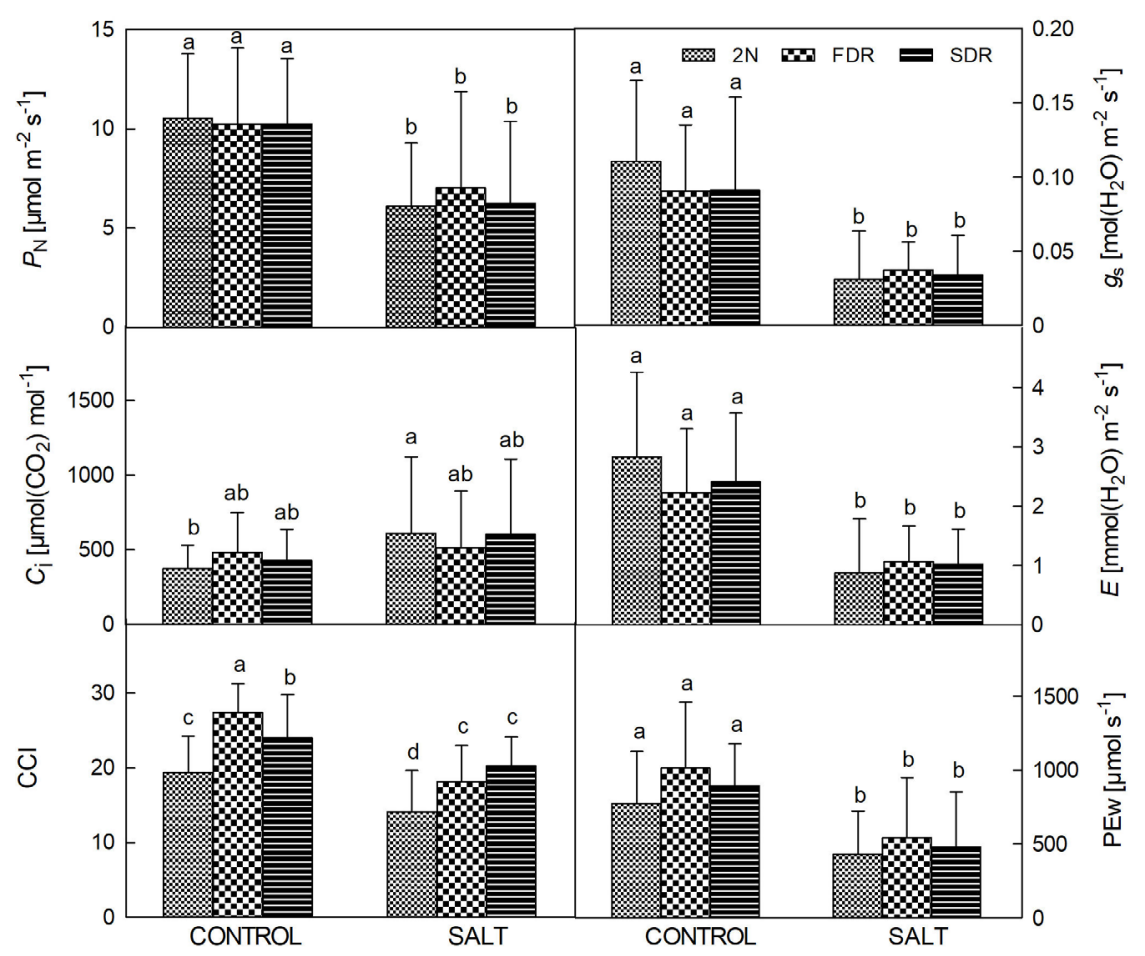

Fig. 2. Changes in net photosynthetic rate $\left(P_{\mathrm{N}}\right)$, stomatal conductance $\left(g_{\mathrm{s}}\right)$, intercellular $\mathrm{CO}_{2}$ concentration $\left(C_{\mathrm{i}}\right)$, transpiration rate $(E)$, chlorophyll content index (CCI), and photosynthetic efficiency of the whole leaf $(\mathrm{PEw})$ of the diploid $(2 \mathrm{~N})$ and two allotriploid populations (FDR and SDR) after $30 \mathrm{~d}$ of salt treatment. Mean values $\pm \operatorname{SD}(n=16)$. Values with the same letter are not significantly different according to the Fisher's LSD test $(p<0.05)$. the diploid population was 0.67 , and the variation ranged from $0.02-0.103 \mu \mathrm{mol} \mathrm{s} \mathrm{s}^{-1}$. In addition, the FDR and SDR triploid populations had seven and nine genotypes with average PEw values lesser than those of the diploid, respectively, and the maximum PEw value in the triploid population was greater than that in the diploid population. Moreover, the coefficients of variation of $g_{\mathrm{s}}, C_{\mathrm{i}}, E$, and PEw in the FDR triploid were lower than those in the SDR triploid, but the $P_{\mathrm{N}}$ and CCI values were opposite.

RMP and MDA: For the average performance of the different treatments, under normal culture conditions, there were no significant differences in the RMP and MDA content between allotriploid and diploid (Fig. 3; Table 3S, supplement). The MDA content of the FDR triploid population was slightly lower, with an average value of $58.37 \mathrm{nmol} \mathrm{g}^{-1}$ (FM), which was 3.2\% lower than that of the diploid population. However, after salt treatment, the average RMP values of the triploid and diploid populations increased significantly compared with those of the control group. The average RMP values of the FDR and SDR triploids were significantly higher than those of the diploids, as they were 34.7 and $19.2 \%$, respectively, higher than those of the diploids. The MDA content in triploid and diploid plants did not increase significantly after salt treatment, but the MDA content of the FDR triploid population increased slightly with an average value of $62.76 \mathrm{nmol} \mathrm{g}^{-1}(\mathrm{FM})$, which was significantly higher by $22.7 \%$ than that of the treated diploid population. Under the salt treatment, there was no significant difference in RMP between the FDR and SDR triploids, but the MDA content of the FDR triploids was significantly higher than that of the SDR triploids.

For the individual characteristics of the different groups, under normal culture conditions, the coefficients of variation of RMP in the FDR and SDR triploid populations were equal; they were both 0.26 and greater than those in the diploid populations. The coefficients of variation of MDA content in the FDR and SDR triploids and diploids were $0.17,0.23$, and 0.21 , respectively. After $30 \mathrm{~d}$ of salt stress, the coefficients of variation of RMP in the FDR and SDR triploid populations were 0.37 and 0.32 , respectively, with the variation ranging from 13.9 to $76.7 \%$, while the coefficient of variation of RMP in the diploid population increased to 0.54 from 0.18 of the control group, with the variation ranging from 12.0 to $78.4 \%$. Moreover, the coefficients of variation of MDA content in the FDR and SDR triploids and diploids were 0.14, 0.26, and 0.25, respectively; the variation ranged from 44.86 to 80.34 $\mathrm{nmol} \mathrm{g}^{-1}(\mathrm{FM})$ and from 29.69 to $74.20 \mathrm{nmol} \mathrm{g}^{-1}(\mathrm{FM})$, respectively, in the FDR and SDR triploid populations, while that in the diploid population ranged from 25.87 to $70.00 \mathrm{nmol} \mathrm{g}^{-1}(\mathrm{FM})$. Among the genotypes, G2, G7, and G13 in the FDR population and G12 in the SDR triploid population had a greater MDA content than the individual maximum of the diploid population, but there were seven genotypes in the triploid population with an MDA content lower than the average diploid level (Fig. 4).

POD and Pro: For the average performance of the different treatments, the average POD activity and Pro contents of triploid and diploid poplar were similar and showed no significant difference in the control group, in which the average POD values of the SDR triploid population were slightly lower, $1,158.57 \mathrm{U} \mathrm{g}^{-1}(\mathrm{FM})$, than those of the other populations (Fig. 5; Table 4S, supplement). After salt stress for $30 \mathrm{~d}$, the POD activity in the triploid poplar population increased significantly compared with that of 


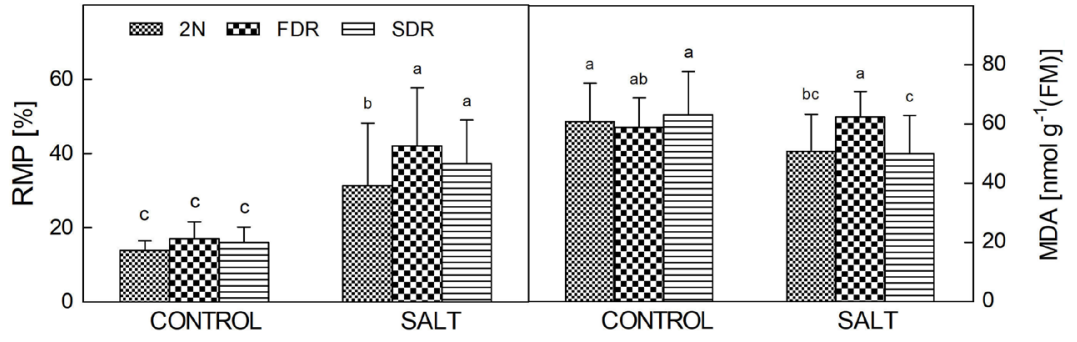

Fig. 3. Changes in relative membrane permeability (RMP) and malondialdehyde (MDA) content of the diploid (2N) and two allotriploid populations (FDR and SDR) after $30 \mathrm{~d}$ of salt treatment. Mean values $\pm \mathrm{SD}(n=16)$. Values with the same letter are not significantly different according to the Fisher's LSD test $(p<0.05)$.

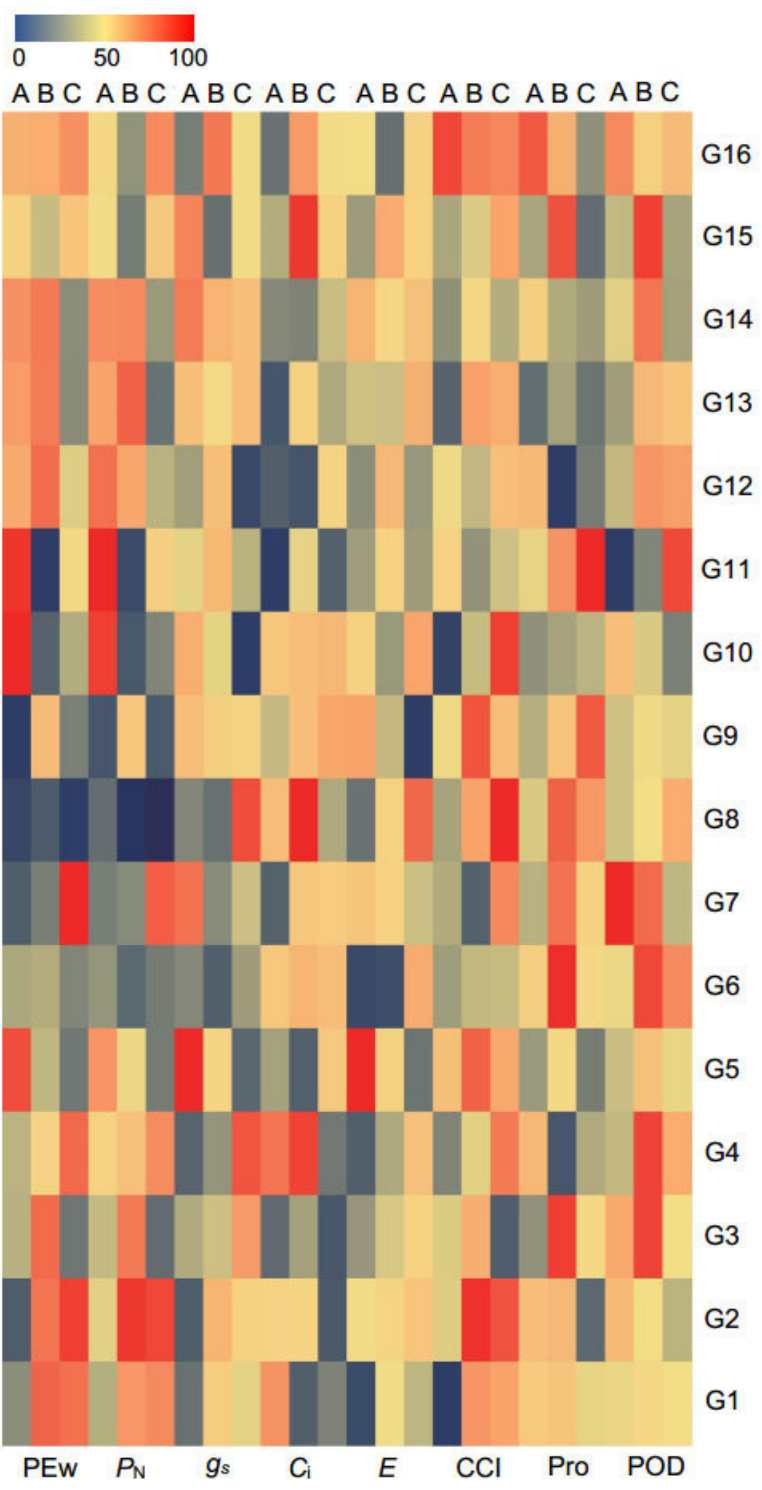

Fig. 4. Changes of $\mathrm{PE}_{\mathrm{w}}, P_{\mathrm{N}}, g_{\mathrm{s}}, C_{\mathrm{i}}, E, \mathrm{CCI}$, Pro and POD in response to salt stress in different populations of Populus cathayana. From left to right, each index has three columns: A, B, C stands for diploid, FDR triploid, and SDR triploid, respectively; from bottom to top, 16 genotypes of three populations, G1-G16, respectively. PEw - photosynthetic efficiency of the whole leaf; $P_{\mathrm{N}}-$ net photosynthetic rate; $g_{\mathrm{s}}-$ stomatal conductance; $C_{\mathrm{i}}-$ intercellular $\mathrm{CO} 2$ concentration; $E$ - transpiration rate; CCI - chlorophyll content index; Pro proline; POD - peroxidase. Red represents a high proportion of all genotypes. Blue represents a low proportion of all genotypes. the control group. The POD activity in the FDR triploid population increased significantly, with an average value of $1,897.96 \mathrm{U} \mathrm{g}^{-1}$, which was significantly higher than that of the diploid population, as it was $36.1 \%$ higher than that of the diploid population. Similarly, the Pro contents in the triploid and diploid populations increased significantly after salt stress. The average Pro content of the FDR triploid population was $105.08 \mu \mathrm{g} \mathrm{g}^{-1}(\mathrm{FM})$, which was significantly higher than that of the diploid, while there was no significant difference in Pro content between the SDR triploid and diploid populations. Moreover, the POD activity and Pro content of the FDR triploid were significantly higher than those of the SDR triploid by 23.7 and $27.7 \%$, respectively.

For the individual characteristics of the different groups, the coefficients of variation of POD activity were 0.25 and 0.40 and the coefficients of variation of Pro content were 0.22 and 0.08 , respectively, in the FDR and SDR triploid populations under normal culture conditions. After the salt stress treatment, compared with the control group, the coefficients of variation of POD activity and Pro content in the triploid and diploid populations increased, and there were significant differences between individuals in the populations. The triploid population had some genotypes whose POD activity and Pro content were lower than the average diploid level. For POD activity, the FDR triploid population had seven genotypes that had values higher than 2,000 $\mathrm{U} \mathrm{g}^{-1}$, while the diploid and SDR triploid populations had two genotypes. For the Pro content, G6 in the FDR and G11 in the SDR triploid population showed the highest values, which were higher than the maximum value of the diploid population. In addition, the trends in variation of POD and Pro in some genotypes were similar; for example, G16 in the diploid population, G3, G6, and G15 in the FDR triploid population and G2, G8, and G11 in the SDR triploid population were all individuals with higher POD and Pro contents in their populations. G5, G9, and G13 in the diploid population and G14 and G15 in the SDR triploid population were all lower POD and Pro individuals in their populations (Fig. 4).

\section{Discussion}

The results showed that a higher concentration of $\mathrm{NaCl}$ solution could increase membrane permeability, lipid peroxidation, and the accumulation of a large amount of MDA in plant leaves (Liu et al. 2008). Under salt stress, plants synthesize antioxidant enzymes, such as peroxidase, to scavenge ROS and accumulate some small molecular osmotic regulators, such as proline, to maintain 


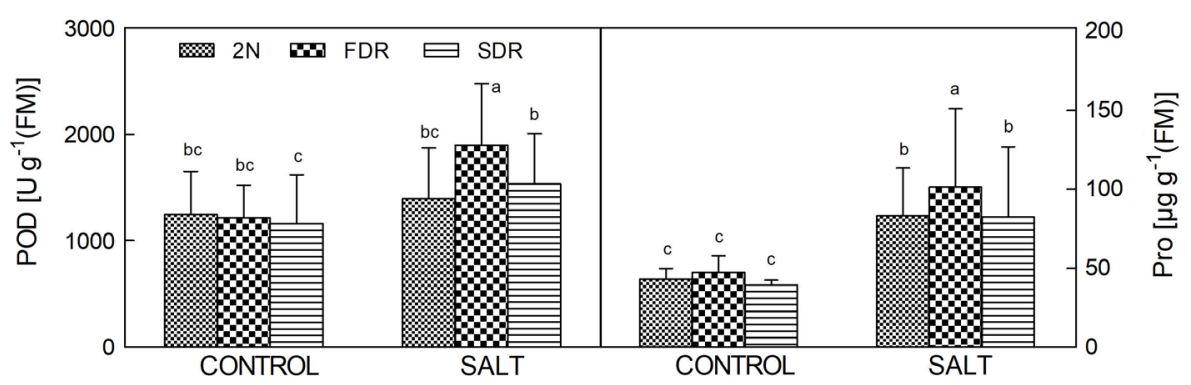

Fig. 5. Changes in peroxidase (POD) activity and proline (Pro) content of the diploid $(2 \mathrm{~N})$ and two allotriploid populations (FDR and SDR) after $30 \mathrm{~d}$ of salt treatment. Mean values \pm $\mathrm{SD}(n=16)$. Values with the same letter are not significantly different according to the Fisher's LSD test $(p<0.05)$. cell osmotic potential under salt stress. (Meloni et al. 2003, Misra and Gupta 2005). Additionally, too much $\mathrm{Na}^{+}$destroys the chloroplast structure and decreases the Chl content and activity of related enzymes leading to the obstruction of primary reaction and carbon assimilation, and the conversion of light energy also decreases (Redondo-Gómez et al. 2010). At the same time, the plants decrease stomatal conductance, reduce water loss, maintain the water balance of the leaves, which is exhibited in the decrease of $g_{\mathrm{s}}$ and $E$, the insufficient supply of $\mathrm{CO}_{2}$, and then the decrease of $P_{\mathrm{N}}$, which all affect plant growth (Shoji et al. 2006, Chastain et al. 2014).

There was no significant difference between the triploid and diploid populations in cell membrane permeability, effect on photosynthesis, antioxidant enzyme activity, and other physiological indexes in the untreated control groups. After salt stress treatment for $30 \mathrm{~d}$, the average RMP value of triploid poplar was significantly higher than that of diploid poplar (Fig. 3), but the triploid poplar produced more antioxidant enzymes and osmotic regulators to resist salt damage (Fig. 5). The triploid poplar population was still higher than the diploid population in terms of the relative growth rate of ground diameter and LA and the photosynthetic efficiency of the whole leaf. The Chl content of triploid poplar was also significantly higher than that of diploid poplar. Therefore, the salt tolerance of the triploid poplar population was higher than that of the diploid poplar population under salt stress, which was consistent with that under normal conditions (Liao et al. 2016).

The growth pattern in plant populations is normally distributed, so there are differences in physiological traits between different individuals in triploid populations (Liao et al. 2017). Salt tolerance is controlled by multiple genes and is characterized quantitatively (Monforte et al. 1997). In this study, there were some differences in all physiological indexes between the 16 genotypes in the triploid and diploid populations, and the coefficient of variation of these indexes ranged from 0.07-1.21. After $30 \mathrm{~d}$ of salt stress, the differences in the 16 genotypes increased, and the coefficient of variation ranged from 0.09-1.70, which showed that the treatment increased the individual differences in salt tolerance. Additionally, the range in PEw variation of the triploid and diploid poplar populations was $0.012-0.158 \mu \mathrm{mol} \mathrm{s} \mathrm{s}^{-1}$ and $0.016-0.103$ $\mu \mathrm{mol} \mathrm{s}{ }^{-1}$, the range in POD variation was $693.03-2,695.50$ $\mathrm{U} \mathrm{g}^{-1}$ and 127.65-2,164.42 $\mathrm{U} \mathrm{g} \mathrm{g}^{-1}$, and the range in Pro variation of the triploid and the diploid was 34.02-193.75 $\mu \mathrm{g} \mathrm{g}^{-1}(\mathrm{FM})$ and $48.79-154.19 \mu \mathrm{g} \mathrm{g}^{-1}$ (FM), respectively.
The results showed that some individuals with a lower salt tolerance in the triploid population were less tolerant than individuals with the high tolerance in the diploid poplar population. However, the individuals with the high salt tolerance in the triploid poplar population were much more tolerant than those in the diploid population. This study also showed that a small population can only explain the differences between the genotypes but that it is difficult to objectively evaluate the overall genetic effects of polyploidy in a comparative study of polyploid and diploid plants. When we compared the differences between different ploidy poplar populations, we also found that there were genotypic differences within the same population, but the triploid was better than the diploid as a whole.

The results showed that the heterozygosity of FDR and SDR were different due to the different pathways of gametogenesis (Lindner et al. 2000, Surtees et al. 2004). FDR transmitted $70-80 \%$ of the parental heterozygosity, but SDR transmitted 30-40\% (Hermsen 1984, Barone et al. 1995). Therefore, it is generally believed that FDR-type triploids exhibit higher heterozygosity and epistasis and have a high utilization value in breeding (Peloquin et al. 2008, Xi et al. 2012). For the triploid of Populus cathayana in the study, FDR and SDR transmitted 74.8 and $39.6 \%$, respectively, of the parental heterozygosity (Dong et al. 2015). The FDR triploid population performed better than the SDR population under normal cultivation conditions (Liao et al. 2017). In this study, the average content of Pro and POD in the FDR triploid population was significantly higher than those in the SDR triploid population after salt stress treatment (Fig. 5). However, the average MDA content in the FDR triploid poplar was also significantly higher than that in the SDR poplar (Fig. 3), and there was no significant difference in RMP between the FDR and SDR triploid populations, suggesting that their relative membrane permeability was similar. Obviously, it was difficult to differentiate the salt tolerance of the triploids from different $2 n$ gametes by cell membrane permeability and ROS indexes. Salt stress could eventually affect the growth and development of the plants, but there was no significant difference in the relative growth rate of $\mathrm{H}$, ground diameter, and LA and the photosynthesis index between the FDR and SDR triploids. The reason may be related to sampling error and homologous recombination (Dong et al. 2014). Hence, the resistance genes may be in the homologous recombination region, and homologous recombination may have enhanced the heterozygosity of the stress resistance genes 
in the SDR triploids, which may have resulted in the salt tolerance of the SDR triploid being comparable to that of the FDR triploid.

\section{References}

Ashraf M.: Relationships between growth and gas exchange characteristics in some salt-tolerant amphidiploid Brassica species in relation to their diploid parents. - Environ. Exp. Bot. 45: 155-163, 2001.

Barone A., Gebhardt C., Frusciante L.: Heterozygosity in 2n gametes of potato evaluated by RFLP markers. - Theor. Appl. Genet. 91: 98-104, 1995.

Castrejón S.E., Yatsimirsky A.K.: Cyclodextrin enhanced fluorimetric determination of malonaldehyde by the thiobarbituric acid method. - Talanta 44: 951-957, 1997.

Chao D.Y., Dilkes B., Luo H. et al.: Polyploids exhibit higher potassium uptake and salinity tolerance in Arabidopsis. Science 341: 658-659, 2013.

Chastain D.R., Snider J.L., Collins G.D. et al.: Water deficit in field-grown Gossypium hirsutum primarily limits net photosynthesis by decreasing stomatal conductance, increasing photorespiration, and increasing the ratio of dark respiration to gross photosynthesis. - J. Plant Physiol. 171: 1576-1785, 2014.

Chen S., Jiang J., Li H.Y., Liu G.F.: The salt-responsive transcriptome of Populus simonii $\times$ Populus nigra via DGE. Gene 504: 203-212, 2012.

Dong C.B., Mao J.F., Suo Y.J. et al.: A strategy for characterization of persistent heteroduplex DNA in higher plants. - Plant J. 80: 282-291, 2014.

Dong C.B., Suo Y.J., Wang J., Kang X.Y.: Analysis of transmission of heterozygosity by $2 \mathrm{n}$ gametes in Populus (Salicaceae). Tree Genet. Genom. 11: 799, 2015.

Godfree R.C., Marshall D.J., Young A.G. et al.: Empirical evidence of fixed and homeostatic patterns of polyploid advantage in a keystone grass exposed to drought and heat stress. - R. Soc. Open Sci. 4: 170934, 2017.

He T., Cramer G.R.: Growth and mineral nutrition of six rapidcycling Brassica species in response to seawater salinity. Plant Soil 139: 285-294, 1992.

Hermsen J.: Mechanisms and genetic implications of $2 \mathrm{n}$ gamete formation. - Iowa State J. Res. 58: 421-434, 1984.

Kumar D.: Salt tolerance in oilseed brassicas - present status and future prospects. - Plant Breed. Abstr. 65: 1438-1447, 1995.

Liao T., Cheng S.P., Zhu X.H. et al.: Effects of triploid status on growth, photosynthesis, and leaf area in Populus. - Trees 30: 1137-1147, 2016.

Liao T., Wang Y., Xu C.P. et al.: Adaptive photosynthetic and physiological responses to drought and rewatering in triploid Populus populations. - Photosynthetica 55: 578-590, 2017.

Lindner K., Seeb J., Habicht C. et al.: Gene-centromere mapping of 312 loci in pink salmon by half-tetrad analysis. - Genome 43: 538-549, 2000.

Liu Y.J., Zhang S.S., Tian X.Y., Ren D.M.: Effects of salt stress on protective enzyme system, MDA content and membrane permeability of NHC foliar. - Grassl. Turf 2: 34-38, 2008.

Luo G., Xue L., Guo R., Lei J.: Creating cold resistant strawberry via interploidy hybridization between octoploid and dodecaploid. - Euphytica 213: 194, 2017.

Meloni D.A., Oliva M.A., Martinez C.A., Cambraia J.: Photosynthesis and activity of superoxide dismutase, peroxidase and glutathione reductase in cotton under salt stress. - Environ. Exp. Bot. 49: 69-76, 2003.

Meng F., Luo Q., Wang Q. et al.: Physiological and proteomic responses to salt stress in chloroplasts of diploid and tetraploid black locust (Robinia pseudoacacia L.). - Sci. Rep.-UK 6: 23098, 2016.

Misra N., Gupta A.K.: Effect of salt stress on proline metabolism in two high yielding genotypes of green gram. - Plant Sci. 169: 331-339, 2005.

Peloquin S.J., Boiteux L.S., Simon P.W., Jansky S.H.: A chromosome-specific estimate of transmission of heterozygosity by $2 \mathrm{n}$ gametes in potato. - J. Hered. 99: 177-181, 2008.

Redondo-Gómez S., Mateos-Naranjo E., Figueroa M.E., Davy A.J.: Salt stimulation of growth and photosynthesis in an extreme halophyte, Arthrocnemum macrostachyum. - Plant Biol. 12: 79-87, 2010.

Segraves K.A., Anneberg T.J.: Species interactions and plant polyploidy. - Am. J. Bot. 103: 1326-1335, 2016.

Shoji T., Suzuki K., Abe T. et al.: Salt stress affects cortical microtubule organization and helical growth in Arabidopsis. Plant Cell Physiol. 47: 1158-1168, 2006.

Sugiyama S.: Responses of shoot growth and survival to water stress gradient in diploid and tetraploid populations of Lolium multiflorum and L. perenne. - Grassl. Sci. 52: 155-160, 2006.

Surtees J.A., Argueso J.L., Alani E.: Mismatch repair proteins: key regulators of genetic recombination. - Cytogenet. Genome Res. 107: 146-159, 2004.

Vieira S.M., Silva T.M., Glória M.B.A.: Influence of processing on the levels of amines and proline and on the physicochemical characteristics of concentrated orange juice. - Food Chem. 119: 7-11, 2010.

Wang J., Li D.L., Kang X.Y.: Induction of unreduced megaspores with high temperature during megasporogenesis in Populus. Ann. For. Sci. 69: 59-67, 2012.

Wang L., Wang Y., Wang X. et al.: Regulation of POD activity by pelargonidin during vegetative growth in radish (Raphanus sativus L.). - Sci. Hortic.-Amsterdam 174: 105-111, 2014.

Wu L., Lin H.: Salt tolerance and salt uptake in diploid and polyploid buffalograsses (Buchloe dactyloides). - J. Plant. Nutr. 17: 1905-1928, 1994.

Xi X.J., Li D., Xu W.T. et al.: 2n egg formation in Populus $\times$ euramericana (Dode) Guinier. - Tree Genet. Genom. 8: 1237-1245, 2012.

Xue H., Zhang F., Zhang Z.H. et al.: Differences in salt tolerance between diploid and autotetraploid apple seedlings exposed to salt stress. - Sci. Hortic.-Amsterdam 190: 24-30, 2015.

Yang C.W., Zhao L., Zhang H.K. et al.: Evolution of physiological responses to salt stress in hexaploid wheat. - P. Natl. Acad. Sci. USA 111: 11882-11887, 2014.

Yang F., Xiao X.W., Zhang S. et al.: Salt stress responses in Populus cathayana Rehder. - Plant Sci. 176: 669-677, 2009.

Zhang F., Xue H., Lu X. et al.: Autotetraploidization enhances drought stress tolerance in two apple cultivars. - Trees 29: 1773-1780, 2015.

(C) The authors. This is an open access article distributed under the terms of the Creative Commons BY-NC-ND Licence. 